Astronomy Reports, vol.46, No.1, 2002, pp.16-26.

Translated from Astronomicheskii Zhurnal, vol.79, No.1, 2002, pp. 19-30

\title{
Observations of Stellar Objects at a Shell Boundary in the Star-Forming Complex in the Galaxy IC 1613^
}

\author{
T.A. Lozinskaya ${ }^{1}$, V.P. Arkhipova ${ }^{1}$, A.V. Moiseev², and V.L. Afanasiev² \\ 1 Sternberg Astronomical Institute, Universitetskiǔ pr. 13, Moscow, Russia, 119899 \\ 2 Special Astrophysical Observatory, Nizhniı̌ Arkhyz, Karachai-Cherkessia, Russia, 369167
}

Received May 7, 2001

\begin{abstract}
The single region of ongoing star formation in the galaxy IC 1613 has been observed in order to reveal the nature of compact emission-line objects at the edges of two shells in the complex, identified earlier in H $\alpha$ line images. The continuum images show these compact objects to be stars. Detailed spectroscopic observations of these stars and the surrounding nebulae were carried out with an integral field spectrograph MPFS mounted on the $6 \mathrm{~m}$ telescope of the Special Astrophysical Observatory. The resulting stellar spectra were used to determine the spectral types and luminosity classes of the objects. An Of star we identified is the only object of this spectral type in IC 1613. The results of optical observations of the multi-shell complex are compared to $21 \mathrm{~cm}$ radio observations. The shells harboring the stars at their boundaries constitute the most active part of the star-forming region. There is evidence that shocks have played an important role in the formation of the shells.
\end{abstract}

\section{INTRODUCTION}

Irregular galaxies provide unique opportunities for studies of star formation triggered by the combined effect of stellar winds and supernova explosions in rich stellar groupings. Due to the absence of spiral density waves in these galaxies, the formation of giant multi-shell complexes around stellar groupings can proceed unhindered, enabling completion of the full ecological cycle of interaction between the stellar and gaseous components of giant molecular clouds. For the same reason, irregular galaxies display the longest scale lengths and time scales on which supernovae and stellar winds play a dominant role in the formation of new-generation stars.

IC 1613, a faint dwarf galaxy of the Local Group, provides one of the most striking examples of a giant multishell complex around a group of young stellar associations. The northeastern sector of IC 1613 harbors a prominent giant complex of ionized shells and supershells surrounding several dozen young stellar associations and star clusters. This complex is the sole site of contemporary star formation in the galaxy (see $[1,2]$ and references therein). The complex also includes the only known supernova remnant

Send offprint requests to: T.A. Lozinskaya, e-mail: lozinsk@sai.msu.ru

* Based on observations collected with the $6 \mathrm{~m}$ and $1 \mathrm{~m}$ telescopes of the Special Astrophysical Observatory (SAO) of the Russian Academy of Sciences (RAS), operated under the financial support of the Science Department of Russia (registration number 01-43) in the galaxy (see [3] and references therein). Comparison of the optical and $21 \mathrm{~cm}$ brightness distributions showed that the shells of HII are surrounded by extended shells of HI [4]. The shells of ionized and neutral gas are close to each other, and partially overlap in the plane of the sky. If the sizes of the shells along the line of sight and in the plane of the sky are comparable, this indicates that these shells and supershells are in physical contact with each other. It is currently thought that collisions of massive expanding shells with each other and/or with giant molecular clouds can serve as triggers of gravitational instability and fragmentation in the collision region, leading to the formation of new-generation stars.

Narrow-band $\mathrm{H} \alpha$ images of the region considered taken in 1995 with the $4 \mathrm{~m}$ telescope of the Kitt Peak National Observatory (KPNO) revealed chains of bright, compact emission-line objects located exactly at the edge of the two shells in the complex. The same region hosts association \#17 from the list of Hodges [5] (the eastern part of this feature was later catalogued as association \#25 in the list [2]). However, in our analysis in 1995, we were not able to establish a one-to-one relationship between compact objects and stars. Moreover, the fact that the stellar objects are located exactly along a thin shell-like structure is of considerable interest on its own. This work was motivated primarily by the desire to study the emission spectra and nature of the chain of objects and their possible relationship to triggered star formation.

With this aim in view, we performed photometry and spectroscopy of this region. Section 2 describes the instru- 
Table 1. Log of MPFS spectroscopic observations

\begin{tabular}{|l|c|c|c|c|}
\hline \multirow{2}{*}{ Field } & \multicolumn{2}{|c|}{ Center coordinates } & \multirow{2}{*}{$T_{\exp }, \mathrm{s}$} & $z$ \\
\cline { 2 - 3 } & R.A. $(2000.0)$ & DEC $(2000.0)$ & & \\
\hline Field I & $01^{h} 05^{m} 6.1^{s}$ & $+02^{\circ} 09^{\prime} 34^{\prime \prime}$ & 2700 & $42^{\circ}$ \\
Field II & $01^{h} 05^{m} 5.2^{s}$ & $+02^{\circ} 09^{\prime} 47^{\prime \prime}$ & 2700 & $43^{\circ}$ \\
Field III & $01^{h} 05^{m} 1.8^{s}$ & $+02^{\circ} 09^{\prime} 35^{\prime \prime}$ & 2700 & $48^{\circ}$ \\
\hline
\end{tabular}

ments, observations, and data reduction technique employed. Section 3 describes the overall structure of the multi-shell complex derived from optical and (21cm line) radio observations, and identifies regions that are of greatest interest for detailed spectroscopy. Based on our photometric observations, we show that the compact emission objects in question are stars. Section 4 reports the results of spectroscopic observations of three selected fields made with the integral field spectrograph MPFS mounted on the $6 \mathrm{~m}$ telescope of the SAO RAS. We obtained the spectra of individual stars located at the shell boundaries and estimated their spectral types and luminosity classes. We have determined the distribution of the intensity ratios of the principal lines in the spectra of the surrounding gaseous nebulae, and constructed the gas radial-velocity field based on $\mathrm{H} \beta$ and [SII] line measurements. Section 5 discusses our main results and conclusions.

\section{OBSERVATIONS AND DATA REDUCTION}

\subsection{Photometry}

The shell complex was imaged in two filters on October 4-5, 2000 with the $1 \mathrm{~m}$ Zeiss-1000 telescope of the Special Astrophysical Observatory of the Russian Academy of Sciences in the process of testing the new focal reducer SCORPIO. A description of the reducer and transmission curves of the interference filters used can be found at http://www.sao.ru/ moisav/scorpio/scorpio.html. The reducer is mounted at the Cassegrain focus of the telescope $(F / 13)$, and the total focal ratio of the system was $F / 9$. The spectrograph uses a TK1024 $1024 \times 1024$ CCD as a detector. The system had an angular resolution of $0.52^{\prime \prime} /$ pixel and a field of view of $8 .^{\prime} 9$. We obtained images in two meddle-band interference filters. The filter with a central wavelength of $\lambda_{c}=6620$ $\AA$ and a passband halfwidth of $\Delta \lambda=190 \AA$ coadded the emission in the $\mathrm{H} \alpha$ and $[\mathrm{NII}]$ lines and with the continuum.

To obtain continuum images, we used a filter centered on $\lambda_{c}=6060 \AA$ with a passband halfwidth of $\Delta \lambda=170 \AA$. The total exposure in each filter and the seeing during the observations were $1800 \mathrm{~s}$ and $1.5^{\prime \prime}$ respectively.

After performing standard procedures for CCD frame reduction (bias frame subtraction, flat-field correction, cosmichits removal), we reduced the images to an absolute energy scale using images of the spectrophotometric standard star $\mathrm{BD}+25^{\circ} 4655$ taken on the same night. The reduction to equatorial coordinates was based on field stars, whose positions were adopted from the digital version of the Palomar Sky Survey (http://stdatu.stsci.edu/dss/dss-form.html).

\subsection{Integral Field Spectroscopy}

The spectroscopic observations were made on October 2324, 2000, using the Multipupil Field Spectrograph (MPFS) mounted at the primary focus of the $6 \mathrm{~m}$ telescope of SAO RAS. A description of the spectroscope can be found at http://www.sao.ru/ gafan/devices/mpfs/mpfs_main.htm.

The new spectrograph has a larger field of view, wider spectral range, and higher quantum efficiency than the earlier version of the MPFS [6]. The spectrograph uses a TK1024 $1024 \times 1024 \mathrm{CCD}$ as a detector and enables the spectra of 240 spatial elements (in the form of square lenses) to be taken simultaneously, forming a $16 \times 15$ array on the sky. The angular size of an image element was $1^{\prime \prime}$. A spectrum of the night-sky background $4 .^{\prime} 5$ from the center of the field of view is taken simultaneously. We obtained spectra with a resolution of $8 \AA$ in the range $4350-6850 \AA$. The seeing was about $2^{\prime \prime}$. We observed three areas in the region of the giant shells, whose positions are shown in Fig. 2. Table 1 gives the equatorial coordinates of the field centers, the total exposure times $\mathrm{T}_{\exp }$, and the mean zenith angles $z$ at the time of observation.

The spectroscopic observations were reduced using IDLbased software developed at the SAO Laboratory of Specroscopy and Photometry of Extragalactic Objects. The preliminary data reduction included bias frame subtraction, flat-field correction, cosmic-hits removal, extraction of the individual spectra from the CCD images, and wavelength calibration using the spectrum of a He-Ne-Ar calibration lamp.

We then subtracted the night-sky spectrum from the linearized spectra and converted the observed fluxes to an absolute energy scale using observations of the spectrophotometric standard star Feige 110 (taken immediately before observing the program objects at a zenith distance of $z=50^{\circ}$ ). We adopted the parameters for this standard from the public database

http://www.eso.org/observing/standards/spectra. Our air-mass corrections were based on the mean spectral atmospheric-extinction curve for the Special Astrophysical Observatory given in [7].

We compared the $\mathrm{H} \alpha$ and [NII] line fluxes measured using the MPFS data with those derived from images of star-free shell regions taken with filters. The two methods (spectrophotometric and photometric) yielded line fluxes that agreed to within about $20 \%$, providing an independent estimate of the actual accuracy of our observations.

\section{OVERALL STRUCTURE OF THE MULTI-SHELL COMPLEX IN THE STAR-FORMING REGION}

The multi-shell complex in the northeastern sector of IC 1613 is the most prominent structure seen in narrow-band $\mathrm{H} \alpha$ images of the galaxy [8-12]. This is the place where the overwhelming majority of the galaxy's bright HII regions, shells, and supershells are concentrated. The stellar component of the complex is represented by about twenty stellar associations and star clusters [2, 5].

Figure 1a shows an $\mathrm{H} \alpha$-line image of the multi-shell complex taken in 1995 with the KPNO 4m telescope. The chain of bright, compact emission-line objects located exactly at the bright rim of the shell (indicated by an arrow) and several compact objects at the edges of other shells are clearly visible. 


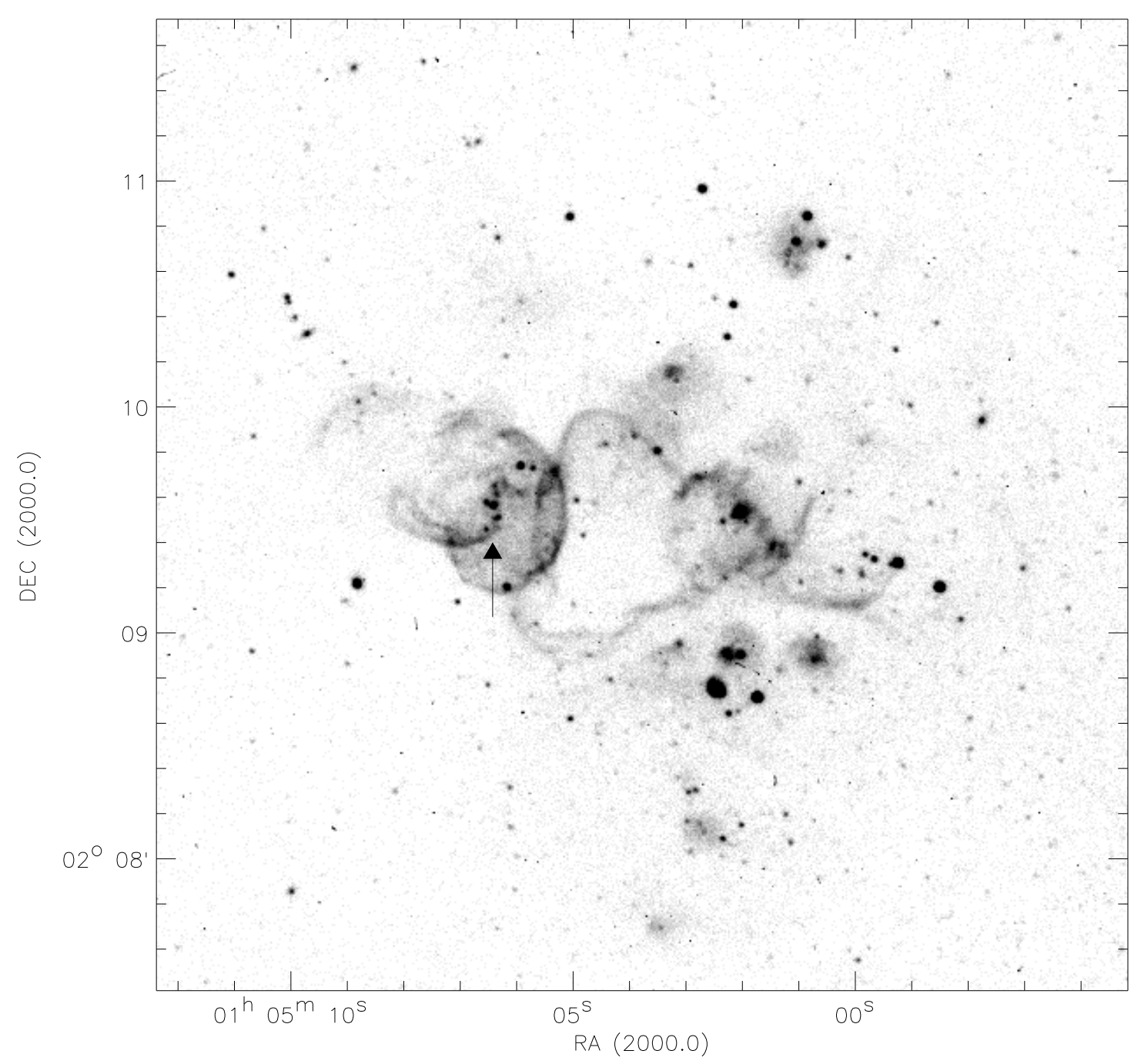

Fig. 1. (a) $\mathrm{H} \alpha$ image of the multi-shell complex taken with the $4 \mathrm{~m}$ telescope of the Kitt Peak National Observatory. The arrow shows the chain of bright, compact emission objects at the shell boundary. (b, see color figure) HI brightness distribution (blue) superimposed on a narrow-band $\mathrm{H} \alpha$ image of the same area (yellow) for the northeastern sector of the galaxy IC 1613 (fragment of a chart published in [4]).

The two shells with compact objects at their boundaries correspond to objects R1 and R2 in the list of shells [12]. Both shells are located within square N27 in Fig. 3 of [12], and include the nebulae S10 and S13 in the classification of Sandage [13].

Meaburn et al. [1] were the first to analyze the kinematics of the complex of ionized shells. Their five spectrograms densely covered the bright part of the complex, enabling determination of the characteristic shell expansion velocities, which proved to be about $30 \mathrm{~km} \mathrm{~s}^{-1}$. Valdez-Gutierrez et al. [12] constructed the line-of-sight velocity field of the entire complex in the $\mathrm{H} \alpha$ and [SII] lines and estimated the expansion velocities of its constituent shells and supershells. HI observations of IC 1613 [14] showed that the complex is located in the region of the brightest "spot" in the $21 \mathrm{~cm}$ radio emission.
Lozinskaya et al. [4] were the first to make a detailed comparison of the $\mathrm{H} \alpha$ and $21 \mathrm{~cm}$ radio brightness distributions of the complex. Figure 1b shows a fragment of the map of the northeastern sector of the galaxy published in [4], with the $\mathrm{HI}$ brightness distribution superimposed on the $\mathrm{H} \alpha$ line image shown in Fig. 1a.

An HI map with a high angular resolution of $7 .^{\prime \prime} 4 \times 7 .^{\prime \prime} 0$ (corresponding to a linear resolution of $\simeq 25 \mathrm{pc}$ ) was constructed using $21 \mathrm{~cm}$ VLA observations (part of a large project to analyze the structure and kinematics of the neutral gas in IC 1613 [15]).

We identified the chain of compact emission-line objects and a number of other compact objects at the edges of the shells shown in Fig. 1a in our $\mathrm{H} \alpha$ line image. These compact emission-line features could be either stars located inside com- 


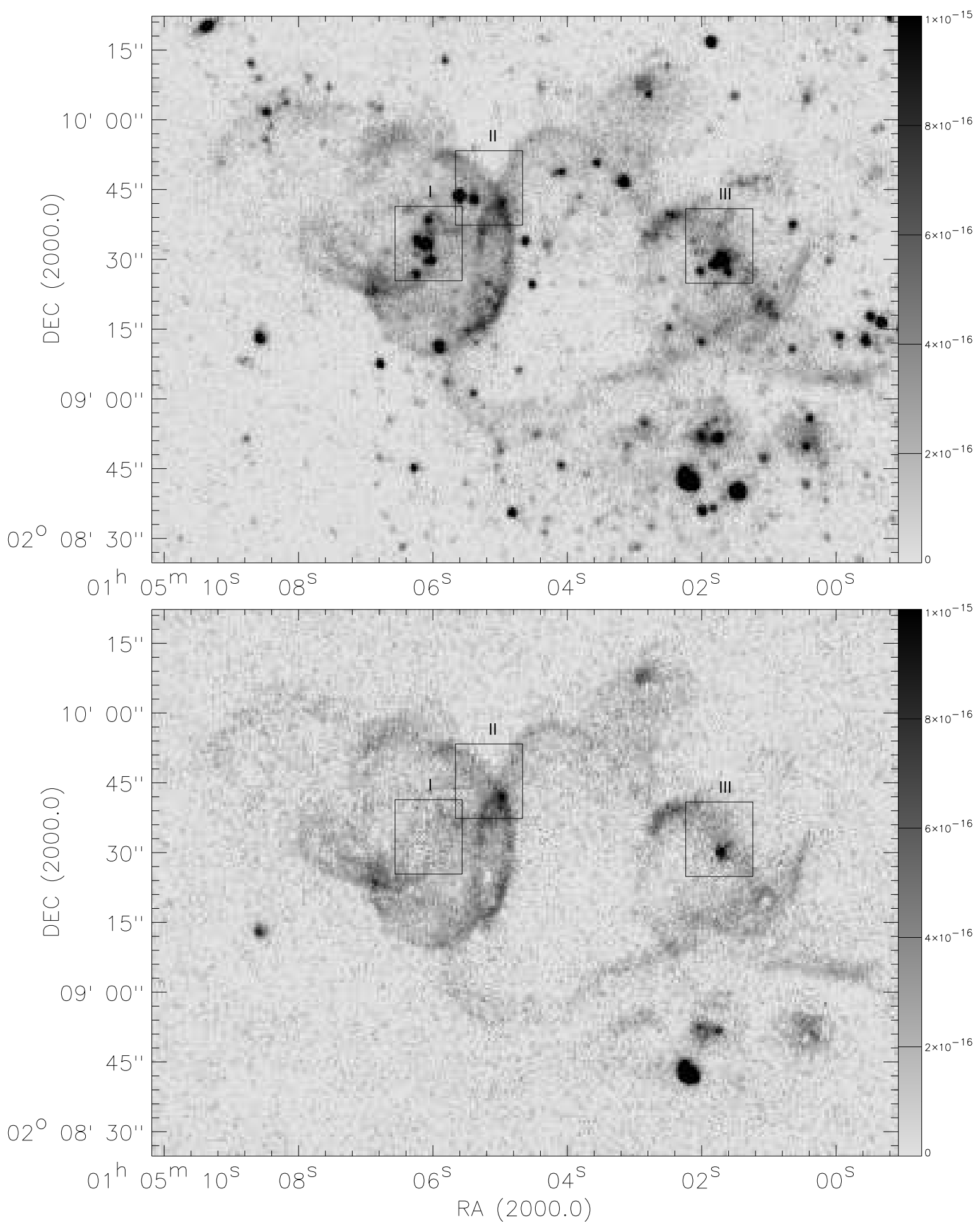

Fig. 2. Image of the complex of ionized shells taken with the Zeiss-1000 telescope equipped with the SCORPIO focal reducer. The scale is in $\mathrm{erg} \cdot \mathrm{s}^{-1} \cdot \mathrm{cm}^{2} / \operatorname{arcsec}^{2}$. Top: image taken with a filter centered on $6620 \AA$ and a passband halfwidth of $190 \AA$ coadding emission in the $\mathrm{H} \alpha$ and [NII] lines and the continuum. Bottom: emission-line image (continuum subtracted). The rectangles indicate regions observed with the SAO 6m telescope using the MPFS.

pact HII regions or dense clumps of gaseous shell material at the initial stage of gravitational fragmentaion and/or shockinduced compression. To elucidate the nature of these compact objects, we performed photometry of the multi-shell complex using the Zeiss-1000 telescope equipped with the SCORPIO focal reducer. 
The upper part of Fig. 2 shows the image of the complex obtained through a filter centered at $6620 \AA$ with a halfwidth of $190 \AA$. This image coadds the emission in the $\mathrm{H} \alpha$ and [NII] lines and the continuum. The lower part of Fig. 2 shows the same image after subtraction of the stellar continuum. To determine the continuum level, we used a filter with maximum sensitivity at $6060 \AA$ and a bandpass halfwidth of $170 \AA$; the shape of the filter transmission curve was rectangular rather than Gaussian. We chose the coefficient for continuum subtraction to ensure the best subtraction of foreground stars. Since the central wavelengths of the two filters differ by almost $600 \AA$, the fact that the slope of the continuum differs from star to star becomes important. This is why many young stars inside the shell complex appear "oversubtracted," due to the appreciable slopes of their continuum (see Section 4). The corresponding locations are masked in Fig. 2.

A comparison of Figs. 1 and 2 suggests that the compact objects can be neither purely gaseous clumps nor stars located inside compact HII regions.

The squares in Fig. 2 indicate Fields I, II, and III for which the spectroscopic observations reported in the next section were made. Figures 3, 4, and 5 show enlargened images of Fields I, II, and III, respectively, obtained with the SCORPIO focal reducer with a filter centered on $6060 \AA$ and a passband halfwidth of $170 \AA$. These continuum images of the three fields indicate that the compact, emission-line objects in question are stars.

We can see 13 stars in Field I, two of which are at the edge of the field. The stars in Field I belong to association \#25 in the list of [2] (the eastern part of association \#17 from [5]). The brightest of these stars are, indeed, located along the bright rim of the shell. We can also see two star-like objects at the boundary of the ionized shell in Field II. Part of association \#17 from [5] is located in this same place. Field III contains stars from association \#13 from the list of Hodge (1978) (\#18 in $[2])$.

Below, we report the results of spectroscopic observations of the brightest of these stars made with a spectrograph MPFS.

\section{RESULTS OF SPECTROSCOPIC OBSERVATIONS}

\subsection{Analysis of Integral Field Spectroscopy}

We fitted Gaussians to emission-line profiles to construct a series of monochromatic images of the fields in the $\mathrm{H} \alpha$ and $\mathrm{H} \beta$ lines and in the [OIII] $5007 \AA$ and [SII] $6717 / 6731 \AA$ forbidden lines. All the line profiles can be adequately fitted by a single Gaussian, without any systematic deviations. Note, however, that our spectral resolution $\left(350-450 \mathrm{~km} \mathrm{~s}^{-1}\right)$ substantially exceeds the expected velocities of relative gas motions [12]. In addition, we also constructed images of the program fields in the stellar continuum at wavelengths $4600-4800 \AA$. Comparison of these data with the continuum images obtained with the Zeiss-1000 telescope enabled us to coadd the spectra from the spatial elements corresponding to individual stars.

Figures 3, 4, and 5 show the results obtained for Fields I, II, and III, respectively (continuum and emission-line field images and the spectra of individual stars with their surrounding nebulae).

\subsubsection{Field I}

We analyzed the spectra of five stars in Field I to estimate their spectral types. Unfortunately, the low resolution and fairly low signal-to-noise ratio prevented us from reliably detecting all spectral features. In addition, superimposed emission lines from ionized gas in the vicinity of the stars can be seen in all the spectra.

Star I.1 can be classified as an O supergiant, judging from the HeII 4541, $4686 \AA$ and SiIII $4552 \AA$ lines in its spectrum.

Star I.2 is evidently much hotter than star I.1, since it exhibits well-defined SiIV 4631, $4654 \AA$ lines; the intensity of its HeII 4686 Åline indicates that the star is a giant rather than a supergiant.

No clearly detectable absorption lines could be found in the spectra of stars I.3 and I.4.

The spectrum of star I.5 exhibits a strong HeII $4686 \AA$ line, indicating the it is an $\mathrm{O}$ star of luminosity class III-V.

We estimated the $B$ and $V$ magnitudes of the stars in the $U B V$ system from their absolute spectral energy distributions, based on the calibration of [16]. The results are summarized in columns $1-3$ of Table 2 . Column 4 gives the $E(B-V)$ color excesses of the stars derived from the $\mathrm{H} \alpha / \mathrm{H} \beta$ Balmer decrements of the nearest HII regions located within Field I. Columns 5, 6 , and 7 give the $(B-V)_{o}$ color index, absolute magnitude $M_{v}$, and the spectral type estimated from $(B-V)_{o}$, taking into account the luminosity classes estimated directly from the spectra.

All estimates in this paper are based on the adopted distance modulus of $(M-m)_{o}=24.31$, corresponding to a distance of $730 \mathrm{kpc}$, in accordance with the new determination [17].

The hottest star in Field I appears to be star I.5.

The emission-line spectra of the gaseous environments of each star are typical of HII regions. The emission-line ratios in the neighborhoods of stars I.1, I.2, I.3, and I.4 are $I([\mathrm{OIII}] 5007 / \mathrm{H} \beta=0.8$; the relative intensities of the same lines for star I.5 yield a ratio of 1.05 , confirming the higher temperature of this star.

Note that all five stars in Table 2 show appreciable interstellar extinction.

There is another hot star with coordinates $\Delta X=+2$, $\Delta Y=+3$ between stars I.1 and I.3. This star does not show up against the bright nebular background emission in images of Field I based on MPFS data. The [OIII] $5007 \AA$ and H $\alpha$ brightness maps (see Fig. 3) show a well-defined emission maximum at this location. The star can be seen on the KPNO frame as a compact clump of $\mathrm{H} \alpha$ emission, and is clearly visible on the Zeiss-1000 plate. (There are actually two stars between stars 1 and 3 , but only one is coincident with the emission peak). This star has $V=21.3^{m}$; the extinction estimated from the Balmer decrement is low and its temperature is high: the line intensities are $I([\mathrm{OIII}] 5007) / I(\mathrm{H} \alpha) \simeq 0.3$ and $I([\mathrm{SII}]) / I(\mathrm{H} \alpha) \simeq 0.2$. We therefore conclude that the excitation of the HII region surrounding the star is radiative, and not associated with shocks. The star is responsible for exciting both its own surrounding gas and the gas in the vicinity of the cooler star I.3.

We used the Zeiss-1000 images to estimate the $V$ magnitudes of fainter stars in Field I relative to star I.4 (for which Table 2 gives $V=19.8^{m}$ ). The $V$ magnitudes of these stars are $20.9^{m}, 21.5^{m}, 21.6^{m}, 21.3^{m}, 21.5^{m}$, and $21.8^{m}$ in order of decreasing brightness. 


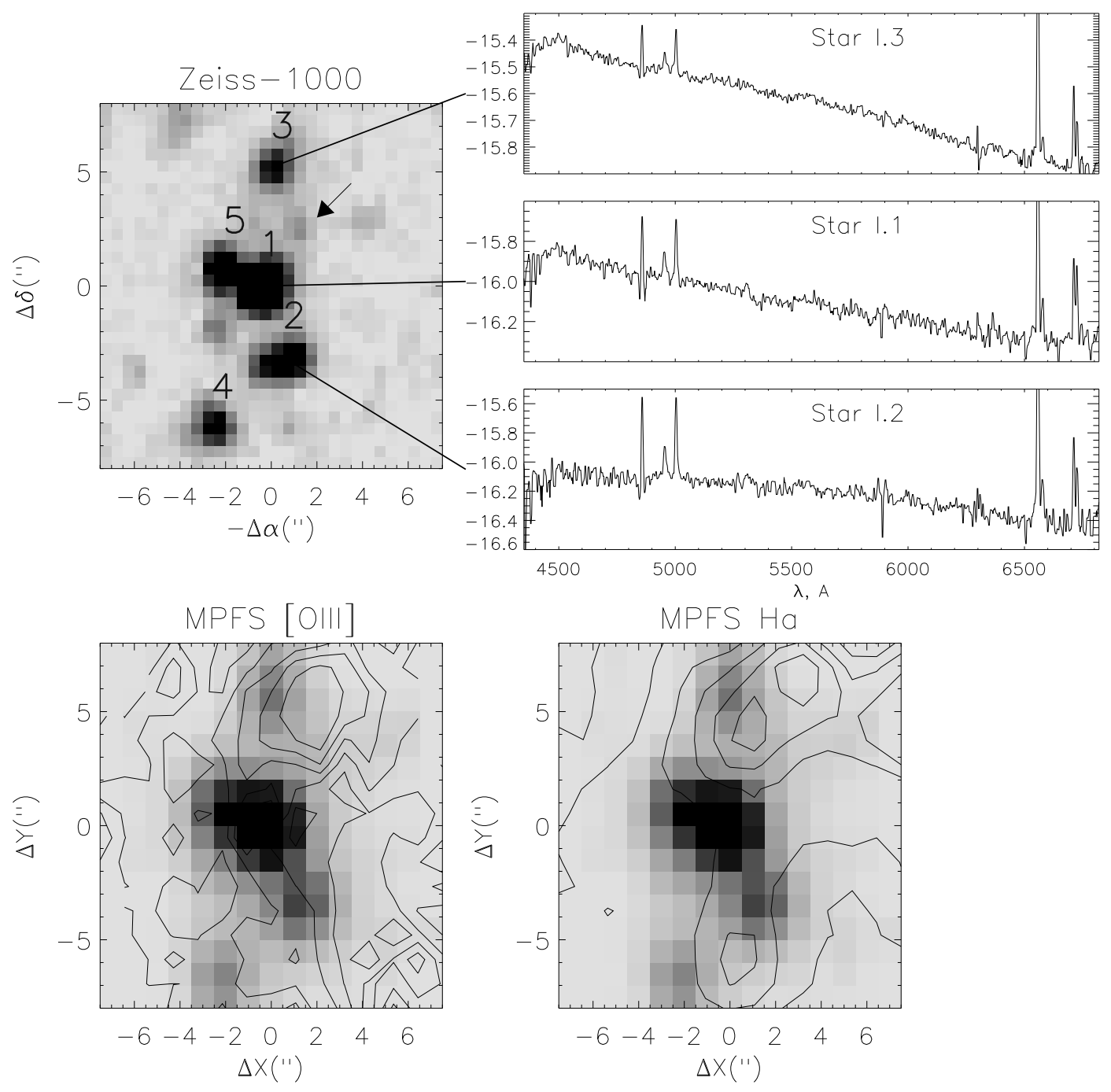

Fig. 3. Top left: Continuum image of Field 1 taken with the Zeiss-1000 telescope through a filter centered on $\lambda_{c}=$ $6060 \AA$ with a passband halfwidth of $\Delta \lambda=170 \AA$. The star numbers are indicated; the arrow marks the hot star that cannot be seen in the MPFS data (see text). Top right: Spectra of the three brightest stars in the field. Bottom: Isophotes of [OIII] (left) and $H \alpha$ (right) line images superimposed on the $4600-4800 \AA$ continuum images (MPFS data).

\subsubsection{Field II}

Our spectra of the two brightest stars in Field II show that these objects are yellow supergiants. We used the same method as for Field I to compute the parameters of these stars presented in Table 3, based on their absolute spectral energy distributions.

The well-localized $\mathrm{H} \alpha$ and [SII] line emission maxima can be seen to the west of star II.2, in the region with $\Delta X=+3$, $\Delta Y=-4$. It is here that the main source of ionizing radiation must be located. However, the emission spectrum of this region is by no means typical of an HII region ionized by an $\mathrm{O}$ star: the relative line intensities are $\mathrm{I}([\mathrm{OIII}] 5007) / \mathrm{I}(\mathrm{H} \alpha)<0.2$ and $\mathrm{I}([\mathrm{SII}]) / \mathrm{I}(\mathrm{H} \alpha) \approx 0.35$. Such parameters are typical of cooling gas behind a shock front.
The electron density of the gas component in this region is low, as indicated by the [SII] intensity ratio of $\mathrm{I}(6717) / \mathrm{I}(6731) \simeq 1.7$, corresponding to $N_{e}<100 \mathrm{~cm}^{-3}$.

Based on the $4600-4800 \AA$ continuum intensity in this region, we estimate the upper limit of the $V$ magnitude of the possible ionizing star to be $19.3^{m}$. This candidate exciting star can be seen at $\Delta X=+6, \Delta Y=-4$ on the map obtained with the Zeiss-1000 telescope.

Note that the line-intensity ratio is $I([\mathrm{SII}]) / I(\mathrm{H} \alpha) \approx 0.35$ virtually throughout the whole of Field II, as is typical of shock excitation. The same field contains part of the shell R2.

\subsubsection{Field III}

We obtained the spectra of seven stars in Field III. Table 4 summarizes the results of our photometric and spectral analy- 


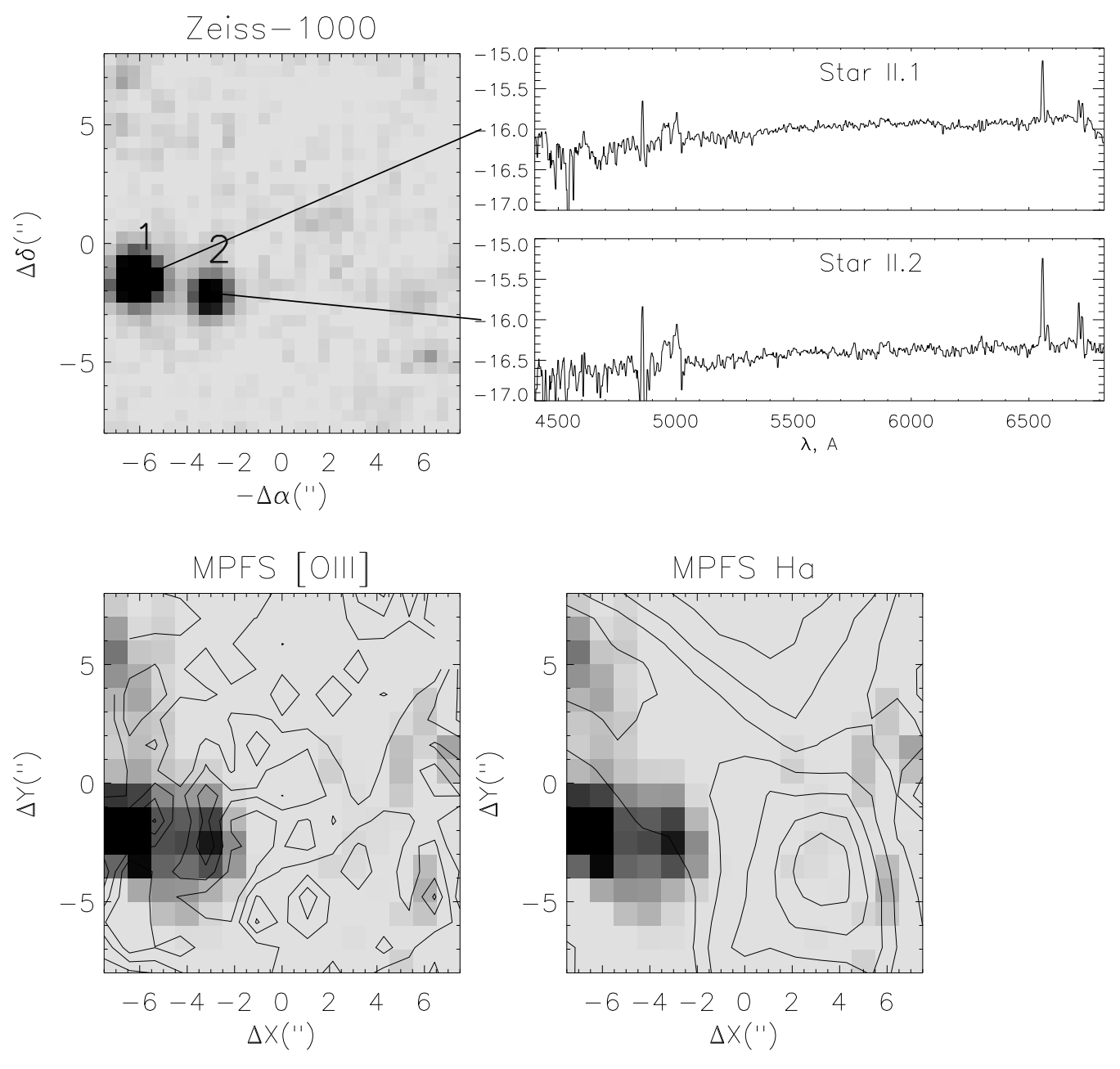

Fig. 4. Same as Fig. 3 for Field II.

sis for these stars. The color indices were determined from the Balmer decrements of HII regions surrounding the stars.

The spectrum of star III.1 appears to show the HeII 4541 $\AA$ line, whereas the HeII $4686 \AA$ line cannot be identified. We conclude that this star is not an $\mathrm{O}$ supergiant, but instead a star with luminosity class III. We cannot, however, rule out the possibility that this might be an early $\mathrm{O}$ main-sequence star.

The spectrum of star III.2 exhibits a strong [OIII] 5007 $\AA$ nebular line: $I([\mathrm{OIII}]) / I(\mathrm{H} \alpha) \simeq 0.4$. This may indicate a high temperature for the exciting star $\left(T \geq 3 \times 10^{4} \mathrm{~K}\right)$. On the other hand, the spectrum of star III.2 may contain HeI 4713 $\AA$ line absorption, as is typical of early B stars. This star could well be binary.

The spectrum of star III.3 shows a well-defined emission band at 4640-4650 $\AA$, typical of WR and Of stars. However, the absence of other lines characteristic of WR stars leads us to conclude that III.3 should be classified as an Of star.

The spectrum of star III.4 shows well-defined HeII 4541 and $4686 \AA$ lines, and possibly SiIV 4630 and $4656 \AA$ lines as well. We classify III.4 a an O7-O8 star of luminosity class III.
Following are the only conclusions we can draw about the three fainter stars in Table 4.

Star III.5 must be blue: its spectrum exhibits well-defined traces of an He $4686 \AA$ absorption line, and its estimated $B-V$ color index is indicative of a high temperature.

Stars III.6 and III.7 must be red, judging from their spectral energy distributions.

The main sources of gas excitation in Field III are stars III. 1 and III.4, and probably also III.2 and III.3. The $\mathrm{H} \alpha$ line emission is concentrated primarily between stars III.1 and III.4, closer to III.4. The [OIII] line emission surrounds star III.4, suggesting that this is the hottest star in the region. According to our estimates, the size of the [OIII] emission region is no less than $6^{\prime \prime}$, corresponding to a linear radius of $R=10 \mathrm{pc}$. The radius of the $\mathrm{H} \alpha$ emission-line region is also about $10 \mathrm{pc}$.

The [SII] line intensity ratio in this region is $I(6717) / I(6731) \simeq 1.5$, corresponding to $N_{e} \leq 100 \mathrm{~cm}^{-3}$.

We thus conclude that all four bright stars in Field III are O stars, without a single supergiant among them. In contrast to Field I, all stars in Field III are giants according to their luminosity classes. 


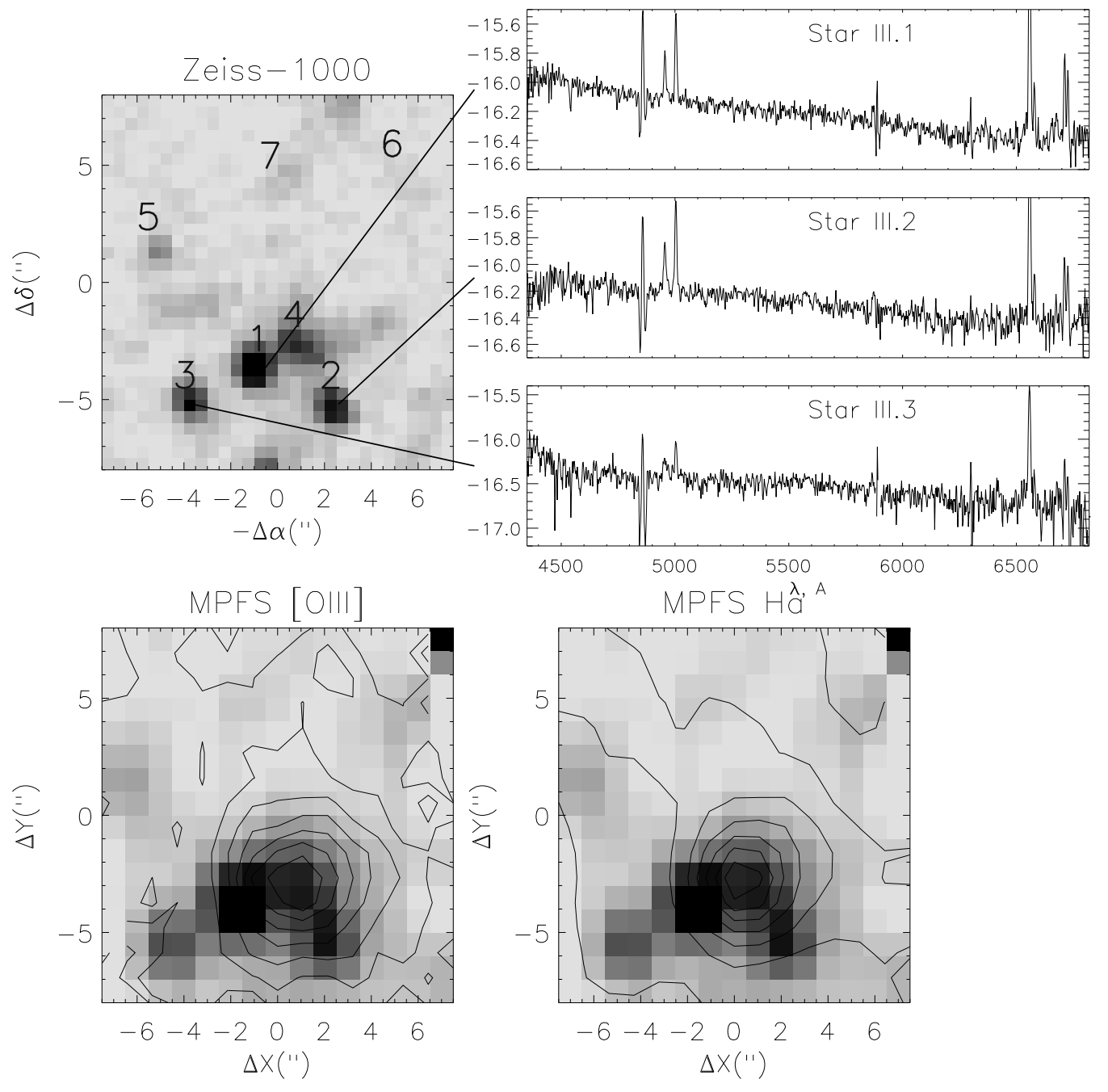

Fig. 5. Same as Fig. 3 for Field III.

\subsection{Ionized Gas Velocities}

Our spectroscopic observations in the $\mathrm{H} \alpha$, [SII], and [OIII] emission lines can be used to construct the line-of-sight velocity fields of the ionized gas in Fields I, II and III. The spectral resolution of our observations is $350-450 \mathrm{~km} \mathrm{~s}^{-1}$, much lower than that of interferometric observations [12]; we therefore do not discuss the results of our velocity measurements in detail here, pointing out only our main conclusions. We found systematic deviations of the order of $20-40 \mathrm{~km} \mathrm{~s}^{-1}$ from the mean value of $V(\mathrm{Hel}) \sim-246 \mathrm{~km} \mathrm{~s}^{-1}$ in each of the three fields. The velocities based on the hydrogen, sulfur, and oxygen line measurements made in the same region vary within the same range. The velocity variations within the regions studied do not go beyond the interval $V(\mathrm{Hel}) \simeq-200$ to $-300 \mathrm{~km} \mathrm{~s}^{-1}$. (Note that, here, we refer to the velocities of the line maxima, not those of weak features).

All the velocity variations in Fields I, II, and III indicated above are in full agreement with the results of interferometric observations of the complex [12].

\section{CONCLUSIONS}

We obtained new observations of the only complex with ongoing star formation in the galaxy IC 1613 to elucidate the nature of the chain of compact emission-line objects we had identified earlier at the peripheries of two shells of the complex in our analysis of deep $\mathrm{H} \alpha$ images. Our new continuum images obtained with the Zeiss-1000 telescope have shown that these compact objects at the boundaries of two shells are stars.

We performed integral field spectroscopy of selected fields in the region of the complex using the MPFS spectrograph mounted on the $6 \mathrm{~m}$ telescope of the Special Astrophysical Observatory. We obtained spectra of the stars forming the chains and estimated their spectral types and luminosity classes. The stars in question are at different evolutionary stages.

Four of the stars forming the chain in Field I at the boundary of the shell R1 are OB stars (with luminosity classes I, II, and III), while one is a cooler object of spectral type A7 III. The position of this last star deviates somewhat from the regular chain structure; we believe that this A7 star is unlikely 
Table 2. Parameters of stars in Field I

\begin{tabular}{|c|c|c|c|c|c|l}
\hline Star & $V$ & $B-V$ & $E(B-V)$ & $(B-V)_{o}$ & $M_{v}$ & \multicolumn{1}{|c|}{ Sp } \\
\hline 1 & 2 & 3 & 4 & 5 & 6 & \multicolumn{1}{|c|}{7} \\
\hline I.1 & 17.94 & +0.02 & 0.33 & -0.31 & -7.4 & OI \\
I.2 & 19.18 & -0.10 & 0.18 & -0.28 & -5.7 & OIII \\
I.3 & 19.32 & +0.37 & 0.18 & +0.19 & -5.5 & A7 III \\
I.4 & 19.80 & +0.10 & 0.18 & -0.08 & -5.0 & B8 II \\
I.5 & 19.80 & -0.05 & 0.29 & -0.30 & -5.4 & O8III or O4V \\
\hline
\end{tabular}

Table 3. Parameters of stars in Field II

\begin{tabular}{|c|c|c|c|c|c|c|}
\hline Star & $V$ & $B-V$ & $E(B-V)$ & $(B-V)_{o}$ & $M_{v}$ & \multicolumn{1}{|c|}{ Sp } \\
\hline II.1 & 18.94 & +1.33 & 0.08 & +1.25 & -5.6 & G8-K0 II \\
II.2 & 20.04 & +1.53 & 0.18 & +1.35 & -4.8 & K0-K2 II-I \\
\hline
\end{tabular}

Table 4. Parameters of stars in Field III

\begin{tabular}{|l|l|l|c|c|c|l|}
\hline Star & \multicolumn{1}{|c|}{$V$} & $B-V$ & $E(B-V)$ & $(B-V)_{o}$ & $M_{v}$ & \multicolumn{1}{|c|}{ Sp } \\
\hline III.1 & 19.40 & +0.01 & $(0.3)$ & -0.29 & $(-5.8)$ & OIII \\
III.2 & 19.62 & +0.07 & 0.3 & -0.23 & -5.6 & O-B \\
III.3 & 20.17 & -0.06 & 0.3 & -0.36 & -5.0 & Of ? \\
III.4 & 19.69 & +0.14 & $(0.4)$ & -0.26 & -5.8 & OIII \\
III.5 & 21.2 & ¡ $0 ?$ & & & & possibly O \\
III.6 & 21.24 & red & & & & \\
III.7 & 21.07 & red & & & & \\
\hline
\end{tabular}

to be a member of the chain, and is simply projected onto the region.

The two brightest stars in Field II lie at the boundary of the shell R2. Our spectra show that these are yellow supergiants. The two stars do not belong to associations identified in the galaxy.

The stars in Field III do not form any clear chain, but are coincident with the brightest clump of emission at the boundary of shell R5. The four brightest stars of the seven whose spectra we obtained are $\mathrm{O}$ stars. The luminosity classes of all these stars indicates that they are giants, with no supergiants among them, in contrast to what we found in Fields I and II. We assigned a spectral type of Of to star III.3. This star, with coordinates $\mathrm{RA}(2000)=1^{\mathrm{h}} 05^{\mathrm{m}} 2 .^{\mathrm{s}} 2$, DEC $(2000)=$ $+02^{\circ} 09^{\prime} 28.6^{\prime \prime}$, is the only star of this type identified in IC 1613 (its coordinates have been measured to within an accuracy of $\left.1^{\prime \prime}\right)$.

One of the three fainter stars must also be of type $\mathrm{O}$; the other two appear to be cooler.

We have identified sources of ionization of the ambient gas in each of the fields considered.

The shells R1 and R2, at whose boundaries the chain in Field I and two stars of Field II are located, stand out among other objects of the multi-shell complex in a number of ways.

(1) These two shells partially overlap in the sky, and form the brightest emission-line region in the entire complex (see Fig. 1). According to estimates made in [12], the $\mathrm{H} \alpha$ luminosities of the two shells are $\log L(\mathrm{H} \alpha)=37.51$ and $\log L(\mathrm{H} \alpha)=$ $37.77 \mathrm{erg} \cdot \mathrm{s}^{-1}$, respectively. The same shells appear to be the brightest in sulfur lines - see Fig. 1 in [12] and Section 4 of this paper. We estimate the line intensity ratio in Field II to be $\mathrm{I}([\mathrm{OIII}] 5007) / \mathrm{I}(\mathrm{H} \alpha)<0.2, \mathrm{I}([\mathrm{SII}]) / \mathrm{I}(\mathrm{H} \alpha) \approx 0.35$, typical of gas fluorescence behind a shock front.

(2) The expansion velocity of shell $\mathrm{R} 2\left(50 \mathrm{~km} \mathrm{~s}^{-1}\right)$ is the highest in the entire complex; the expansion velocity of $\mathrm{R} 1$ is $30 \mathrm{~km} \mathrm{~s}^{-1}$ [12]. Note that Valdez-Gutierres et al.[12] measured both velocities as the mean separation between the two maxima in the double-humped integrated $\mathrm{H} \alpha$ line profiles of the corresponding shells. In fact, images of the complex in hydrogen and sulfur lines in various velocity intervals show both shells to display very complex kinematics. The fact that the brightness of both objects is high even at the extremes of the velocity interval covered --272 and $-177 \mathrm{~km} \mathrm{~s}^{-1}$ - means that the velocities of internal motions within the shells substantially exceed the typical expansion velocities indicated above. Two faint symmetric features in the integrated line profile of shell $\mathrm{R} 2$ with maxima at velocities of -50 and about $-370 \mathrm{~km} \mathrm{~s}^{-1}$ are also immediately apparent (see Fig. 9 in [12]).

By analogy with the results that one of us (TAL) obtained by studying the kinematics of supernova remnants and shells surrounding OB associations, we suggest that these weaker high-velocity line features are closer to the shock-front velocity than brighter low-velocity features. In this case, the shock velocity in the shell should be at least $100 \mathrm{~km} \mathrm{~s}^{-1}$. It is possible that we observe here the same "two-component" gas kinematics as in the Galactic shell surrounding Cyg OB1, Cyg OB3, and a number of other Galactic objects [18], namely the coexistence in a single shell of bright emission at low velocities and weak emission at higher velocities.

Such high internal velocities also reflect the substantial role played by shocks in the formation of both shells at whose boundaries the stellar chains are located.

(3) Comparison of the $21 \mathrm{~cm}$ and $\mathrm{H} \alpha$-line brightness observations in Fig. 1b indicates that the part of the ionized shell R1 where the stellar chain is located falls in the thinnest neutralgas "bridge" connecting the two neutral shell-like structures. The characteristic morphology of the ionized and neutral gas shells indicates that they are in physical contact: the neutral bridge is closely pressed up against the ionized shell R1 from outside in the region of the stellar chain, and the two shells in 
this region have the same radii of curvature. This morphology could be the result of the expanding shell R1 colliding with the neutral bridge located between the two HI shells.

All the facts listed above suggest that the shells R1 and $\mathrm{R} 2$ are located in the most dynamically active part of the starforming complex. This is indeed where new-generation stars are most likely to be born. We plan further detailed high spatialand spectral-resolution studies of the kinematics of the neutral and ionized gas components in this region.

Acknowledgements. This work was supported by the Russian Foundation for Basic Research (project code 01-02-16118) and the Astronomy State Science and Technology Program (project 1.3.1.2). We are grateful to E. Blanton for providing an $\mathrm{H} \alpha$-line image of IC 1613 taken upon our request with the $4 \mathrm{~m}$ telescope of the Kitt Peak National Observatory, to V. N. Komarova for his assistance with the observations at the Zeiss-1000 telescope, and to the Program Committee of the $6 \mathrm{~m}$ telescope for providing observing time.

\section{References}

[1] [1] Meaburn, J., Clayton, C.A. \& Whitehead, M.J., 1988, MNRAS, 479

[2] Georgiev, L., Borissova, J., Rosado, M. et al., 1999, A\&AS, 134,21

[3] Lozinskaya, T.A., Silchenko, O.K., Helfand, D.J. \& Goss, M.W., 1998, AJ, 116, 2328

[4] Lozinskaya, N.A., Moiseev, A.V., Afanas'ev, V.L. et al.,2001, Astron. Rep., 45, 417 astro-ph/0103312

[5] Hodge, P.W., 1978, ApJS, 37, 145

[6] Afanasiev, V.L., Vlasyuk, V.V., Dodonov, S.N. \& Sil'chenko, O.K., 1990, Preprint 54, SAO RAN

[7] Kartashova T.A. \& Chumakova, N.M., 1978, Astrofiz. Issled., 10, 44

[8] Lequeux, J., Meysonnier, N. \& Azzopardi, M., 1987, A\&AS, 67, 169

[9] Hodge, P.W., Lee, M.G. \& and Gurwell, M., 1990, PASP, 102, 1245

[10] Price, J.S., Mason, S.F. \& and Gullixson, C.A., 1990, AJ, 100, 420

[11] Hunter, D.A, Hawley, W.N. \& Gallagher, J.S., 1993, AJ, 106,1797

[12] Valdez-Gutierrez, M., Rosado, M., Georgiev, L. et al., 2001, A\&A, 366, 35

[13] Sandage, A.R., 1971, ApJ, 166, 13

[14] Lake, G., \& Skillman, E.D., 1989, AJ, 98, 1274

[15] Wilcots, E. et al., 2001 in press

[16] Straizys, V., 1992, "Multicolor Stellar Photometry" (Pachart Publ. House, Tucson)

[17] Dolphin, A., Saha, A., Skillman, E.D. et al., 2001, ApJ, 550,554

[18] Lozinskaya, T.A. 1998, Astron. Lett., 24, 237

Translated by A. Dambis 
This figure "fig1b.jpg" is available in "jpg" format from: http://arxiv.org/ps/astro-ph/0112377v1 